\title{
NAtasha Alechina
}

\section{Model checking for coalition announcement logic}

\author{
Natasha Alechina \\ University of Nottingham, \\ Jubilee Campus, Nottingham, NG8 1BB, UK. \\ E-mail: natasha.alechina@nottingham.ac.uk
}

\begin{abstract}
This talk is based on joint work with Rustam Galimullin and Hans van Ditmarsh, published in the German Conference on Artificial Intelligence (KI 2018). First I will introduce background and motivation for the work. I will introduce multi-agent Epistemic Logic (EL) for representing knowledge of (idealised) agents, Public Announcement Logic (PAL) for modelling knowledge change after truthful announcements, Group Announcement Logic (GAL) for modelling what kinds of changes in other agents' knowledge a group of agents can effect, and Coalition Announcement Logic (CAL) which is the main subject of the talk. CAL studies how a group of agents can enforce a certain outcome by making a joint announcement, regardless of any announcements made simultaneously by the opponents. The logic is useful to model imperfect information games with simultaneous moves. It is also useful for devising protocols of announcements that will increase some knowledge of some agents, but also preserve other agents' ignorance with respect to some information (in other words, preserve privacy of the announcers). The main new technical result in the talk is a model checking algorithm for CAL, that is, an algorithm for evaluating a CAL formula in a given finite model. The model-checking problem for CAL is PSPACE-complete, and the protocol requires polynomial space (but exponential time).
\end{abstract}

Keywords: epistemic logic, public announcement logic, coalition announcement logic, model checking algorithm

For citation: Alechina N. "Model checking for coalition announcement logic", Logicheskie Issledovaniya / Logical Investigations, 2018, Vol. 24, No. 2, pp. 59-69. DOI: 10.21146/20741472-2018-24-2-59-69

\section{What this talk is about}

Logics for describing announcements by (groups of) agents, and how announcements affect agents' knowledge. More precisely, model checking algorithm for Coalition Announcement Logic.

The report based on the paper just accepted for the German Conference on Artificial Intelligence (KI 2018).

(C) Alechina N. 
First, we will introduce some background on logic of knowledge, logic of public announcements, and logics of group and coalition announcements.

\subsection{Multi-agent epistemic logic: example}

For more detailed exposition of epistemic logic see [Hintikka, 1962].

Consider the following example: there are three agents, $a, b$ and $c$.

Suppose that, $a$ and $b$ are households that either consume or not consume power ( $p_{1}$ is true if $a$ 's power is on, and $p_{2}$ is true if $b$ 's power is on). $c$ is an electricity substation that needs to know how many households consume power, but not whether individual households consume power or not.

We describe above the situation of $c$ not knowing anything about power consumption (and $a$ and $b$ knowing their own and each other's status).

Possible worlds (or states) are $w_{0}, w_{1}, w_{2}, w_{3}$ with different truth values of $p_{1}$ and $p_{2}$. In $w_{0}, a$ and $b$ know that they are in $w_{0}$ and $\neg p_{1} \wedge p_{2}$ is true $c$ does not know whether $p_{1}$ and $p_{2}$ are true (see Fig. 1):

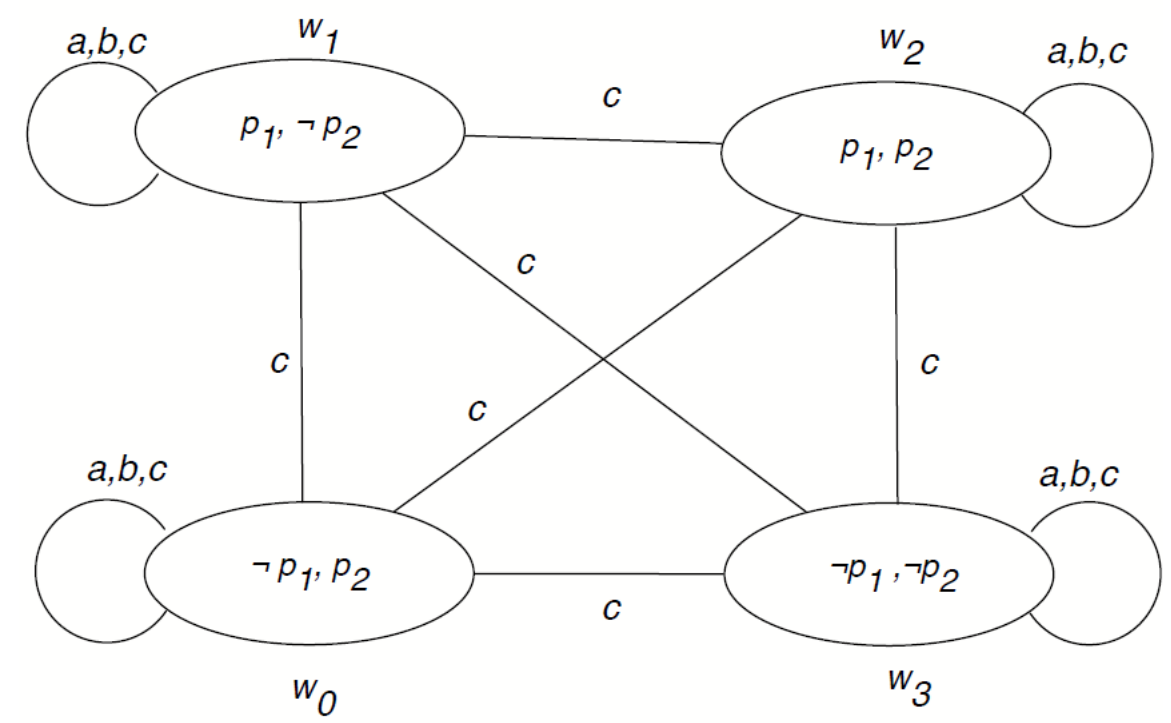

Fig. 1. Example 1.

\section{Multi-agent epistemic logic}

Fix a non-empty finite set of agents $A$ and a set of propositional variables $P$.

Definition 1. Kripke model A Kripke model is a triple $M=(W, \sim, V)$, where

- $W$ is a non-empty set of states,

- $\sim: A \rightarrow \mathcal{P}(W \times W)$ assigns an equivalence relation to each agent, and 
- $V: P \rightarrow \mathcal{P}(W)$ assigns a set of states to each propositional variable.

- $M$ is called finite, if $W$ is finite.

- A pair $(M, w)$ with $w \in W$ is called a pointed model, where $w \in W$ is an actual world.

Consider the following epistemic language $\mathcal{L}_{E L}$ :

$$
\varphi, \psi::=p|\neg \varphi|(\varphi \wedge \psi) \mid K_{a} \varphi
$$

where $p \in P, a \in A$, and all the usual abbreviations of propositional logic and conventions for omitting parentheses hold. $K_{a} \varphi$ stands for ' $a$ knows that $\varphi$ '. The dual operator ' $a$ considers $\varphi$ possible' $\widehat{K}_{a} \varphi$ is defined as $\neg K_{a} \neg \varphi$.

Forcing relation is defined as follows:

$$
\begin{array}{lll}
(M, w)=p & \text { iff } & w \in V(p) \\
(M, w)=\neg \varphi & \text { iff } & (M, w) \not \models \\
(M, w)=\varphi \wedge \psi & \text { iff } & (M, w) \models \varphi \text { and }(M, w) \models \psi \\
(M, w)=K_{a} \varphi & \text { iff } & \forall v \in W: w \sim_{a} v \operatorname{implies}(M, v) \models \varphi
\end{array}
$$

Let us consider the following example:

$$
\begin{aligned}
& \left(M, w_{0}\right) \models K_{a}\left(\neg p_{1} \wedge p_{2}\right) \\
& \left(M, w_{0}\right) \models \neg K_{c}\left(\neg p_{1} \wedge p_{2}\right) \\
& \left(M, w_{0}\right) \models \neg K_{c} \neg p_{1}
\end{aligned}
$$

See the Example 1 on Fig. 1.

\section{Public Announcement Logic (PAL)}

Public announcement logic was initally proposed by Plaza [Plaza, 2007].

Let us consider the following examples:

Suppose $c$ hears that $\left(\neg p_{1} \wedge p_{2}\right) \vee\left(p_{1} \wedge \neg p_{2}\right)$ is true. Some worlds become impossible (see Fig. 2). 


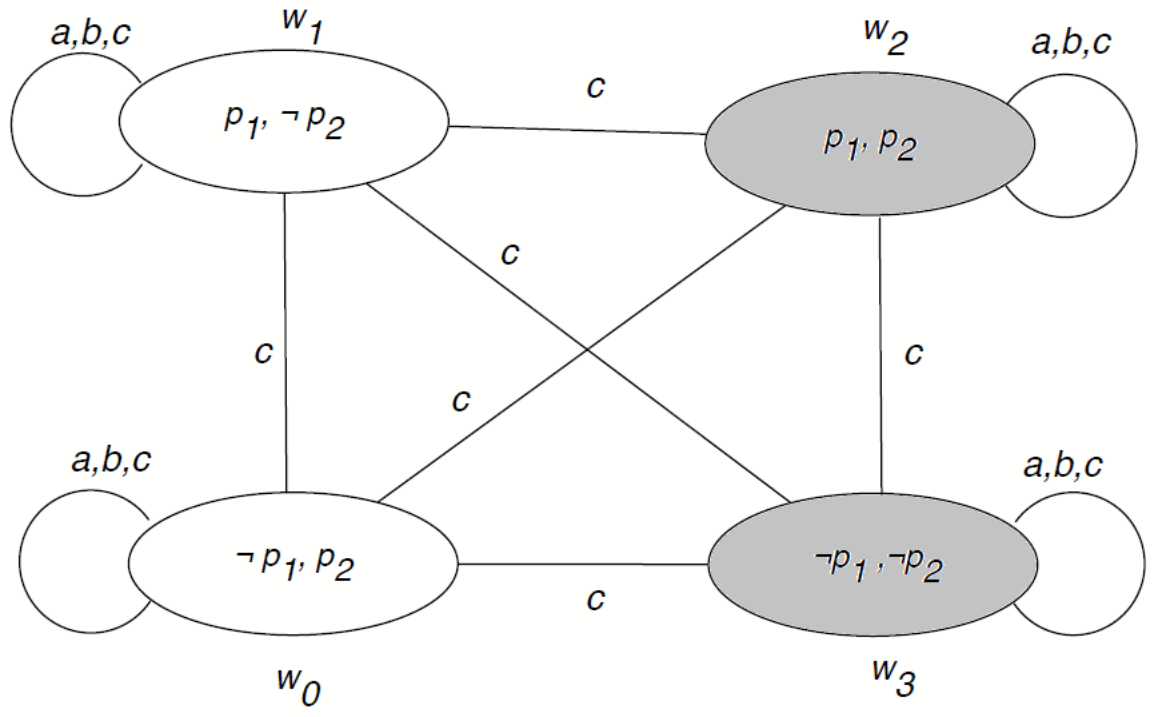

Fig. 2. Example 2.

Suppose $c$ hears that exactly one of the households consumes power: $\left(\neg p_{1} \wedge\right.$ $\left.p_{2}\right) \vee\left(p_{1} \wedge \neg p_{2}\right)$. After this announcement, $c$ only considers $w_{0}$ and $w_{1}$ possible (see Fig. 3).

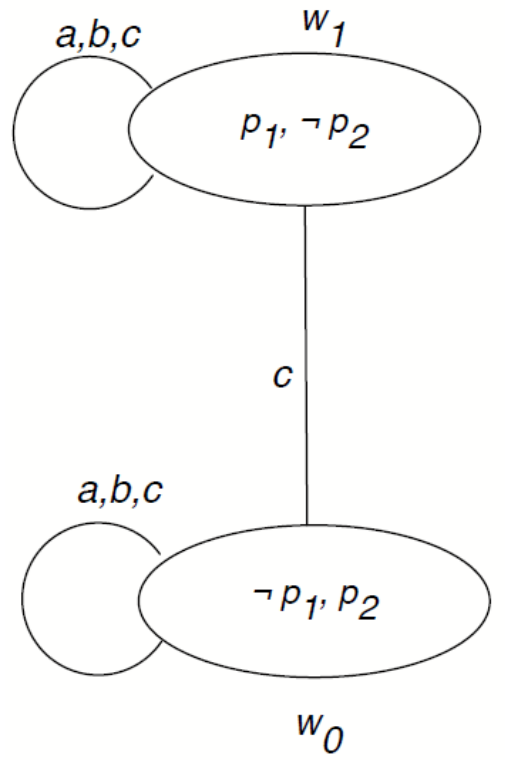

Fig. 3. Example 3. 


\subsection{Definitions}

The language of PAL is $\mathcal{L}_{P A L}$ :

$$
\varphi, \psi::=p|\neg \varphi|(\varphi \wedge \psi)\left|K_{a} \varphi\right|[\psi] \varphi,
$$

where as before, $p \in P, a \in A$.

$[\psi] \varphi$ stands for 'after $\psi$ is truthfully announced, $\varphi$ holds'.

The dual operator $\langle\psi\rangle \varphi$ is defined as $\neg[\psi] \neg \varphi$ and means ' $\psi$ is true, and after it is announced, $\varphi$ is true'.

Given $(M, w)$ and $\varphi \in \mathcal{L}_{E L}$, an updated model $(M, w)^{\varphi}$ is a restriction of the original model to the states where $\varphi$ holds.

$$
\begin{aligned}
& (M, w) \models[\varphi] \psi \quad \text { iff } \quad(M, w) \models \varphi \text { implies }(M, w)^{\varphi} \models \psi \\
& (M, w) \models\langle\varphi\rangle \psi \quad \text { iff } \quad(M, w) \models \varphi \text { and }(M, w)^{\varphi} \models \psi
\end{aligned}
$$

\section{Group and Coalition Announcement Logics (GAL and CAL)}

Intuition: announcements by (group of) agents:

- announcements are made by agents

- agents can only announce what they know

- for example, $a$ can announce $K_{a}\left(\left(\neg p_{1} \wedge p_{2}\right) \vee\left(p_{1} \wedge \neg p_{2}\right)\right)$, but $c$ can not

- the only thing $c$ can announce in $\left(M, w_{0}\right)$ is $K_{c} \top$

- a group of agents can announce a conjunction of formulas, each formula known by an agent in the group.

The language $\mathcal{L}_{G A L}$ of group announcement logic [Ågotnes, van Ditmarsch, $2008]$ is $\mathcal{L}_{P A L}$ extended with $\langle G\rangle \varphi$, where $G \subseteq A$, which stands for 'there is a truthful announcement by $G$, after which $\varphi$ holds'. Let $\mathcal{L}_{E L}^{G}$ denote the set of formulas of the type $\bigwedge_{a \in G} K_{a} \varphi_{a}$, where for every $a \in G$ it holds that $\varphi_{a} \in \mathcal{L}_{E L}$.

$$
(M, w) \models\langle G\rangle \varphi \text { iff } \exists \psi \in \mathcal{L}_{E L}^{G}:(M, w) \models\langle\psi\rangle \varphi
$$

The language $\mathcal{L}_{C A L}[\AA ̊ \AA g o t n e s$ et all, 2010] of coalition announcement logic is $\mathcal{L}_{P A L}$ extended with $\langle[G \rrbracket \varphi$, where $G \subseteq A$, which stands for 'there is an announcement by $G$ such that whatever agents in $A \backslash G$ announce simultaneously, afterwards $\varphi$ holds':

$$
(M, w) \models\left\langle\left[ G \rrbracket \varphi \text { iff } \exists \psi \in \mathcal{L}_{E L}^{G} \forall \chi \in \mathcal{L}_{E L}^{A \backslash G}:(M, w) \models \psi \wedge[\psi \wedge \chi] \varphi\right.\right.
$$


- $a$ and $b$ together can make an announcement after which $K_{c}\left(\left(\left(\neg p_{1} \wedge p_{2}\right) \vee\right.\right.$ $\left.\left.\left(p_{1} \wedge \neg p_{2}\right)\right) \wedge \neg K_{c} \neg p_{1}\right)$ holds: $\left(M, w_{0}\right) \models\langle a, b\rangle K_{c}\left(\left(\left(\neg p_{1} \wedge p_{2}\right) \vee\left(p_{1} \wedge \neg p_{2}\right)\right) \wedge\right.$ $\left.\neg K_{c} \neg p_{1}\right)$; also, $\left(M, w_{0}\right) \models\langle[a, b]\rangle K_{c}\left(\left(\left(\neg p_{1} \wedge p_{2}\right) \vee\left(p_{1} \wedge \neg p_{2}\right)\right) \wedge \neg K_{c} \neg p_{1}\right)$

- $a$ can make an announcement after which $K_{c}\left(\left(\left(\neg p_{1} \wedge p_{2}\right) \vee\left(p_{1} \wedge \neg p_{2}\right)\right) \wedge\right.$ $\left.\neg K_{c} \neg p_{1}\right)$ holds: $\left(M, w_{0}\right) \models\langle a\rangle K_{c}\left(\left(\left(\neg p_{1} \wedge p_{2}\right) \vee\left(p_{1} \wedge \neg p_{2}\right)\right) \wedge \neg K_{c} \neg p_{1}\right)$

- $a$ cannot make an announcement after which $K_{c}\left(\left(\neg p_{1} \wedge p_{2}\right) \vee\left(p_{1} \wedge \neg p_{2}\right)\right) \wedge$ $\left.\neg K_{c} \neg p_{1}\right)$ holds, no matter what other agents announce simultaneously (because $b$ can announce $K_{b} p_{2}$ ):

$$
\left(M, w_{0}\right) \not \models\left\langle[a] K_{c}\left(\left(\left(\neg p_{1} \wedge p_{2}\right) \vee\left(p_{1} \wedge \neg p_{2}\right)\right) \wedge \neg K_{c} \neg p_{1}\right)\right.
$$

\section{Model checking CAL}

Model checking problem for a logic $L$ : given a (finite) model $M$ of $L$ and a formula $\phi$ of $L$, does it hold that $M=\phi$ ?

Model-checking problem for CAL: given a pointed Kripke model $(M, w)$ and a formula $\phi$ of CAL, does it hold that $M \models \phi$ ?

The model checking problem for CAL is interesting because we can use it to plan epistemic actions. What can we tell other agents so that we are guaranteed to get just the right information to them without revealing too much. For example, epistemic planning and verification of distributed protocols.

Definition 2. Bisimulation Let two models $M=(W, \sim V)$ and $M^{\prime}=\left(W^{\prime}, \sim^{\prime}\right.$, $\left.V^{\prime}\right)$ be given. A non-empty binary relation $Z \subseteq W \times W^{\prime}$ is called a bisimulation if and only if for all $w \in W$ and $w^{\prime} \in W^{\prime}$ with $\left(w, w^{\prime}\right) \in Z$ :

- $w$ and $w^{\prime}$ satisfy the same propositional variables;

- for all $a \in A$ and all $v \in W$ : if $w \sim_{a} v$, then there is a $v^{\prime}$ such that $w^{\prime} \sim_{a} v^{\prime}$ and $\left(v, v^{\prime}\right) \in Z$

- for all $a \in A$ and all $v^{\prime} \in W^{\prime}$ : if $w^{\prime} \sim_{a} v^{\prime}$, then there is a $v$ such that $w \sim_{a} v$ and $\left(v, v^{\prime}\right) \in Z$.

Let $(M, w),\left(M^{\prime}, w^{\prime}\right)$ are pointed models and $Z \subseteq W \times W^{\prime}$ is a bisimulation, then $(M, w)$ and $\left(M^{\prime}, w^{\prime}\right)$ are bisimilar.

If $Z_{1}, Z_{2}$ are bisimulations, then $Z_{1} \cup Z_{2}$ is a bisimulation. Union of all bisimulations is a maximal bisimulation.

Definition 3. The quotient model of $M$ with respect to some relation $R$ is $M^{R}=\left(W^{R}, \sim^{R}, V^{R}\right)$, where $W^{R}=\{[w] \mid w \in W\}$ and $[w]=\{v \mid w R v\}$, $[w] \sim_{a}^{R}[v]$ iff $\exists w^{\prime} \in[w], \exists v^{\prime} \in[v]$ such that $w^{\prime} \sim_{a} v^{\prime}$ in $M$, and $[w] \in V^{R}(p)$ iff $\forall w^{\prime} \in[w]: w^{\prime} \in V(p)$. 
Definition 4. Bisimulation contraction of $M$ (written $|M|$ ) is the quotient model of $M$ with respect to the maximal bisimulation of $M$ with itself, i.e. bisimulation contraction is the minimal representation of $\mathrm{M}$.

Definition 5. A model $M$ is bisimulation contracted if $M$ is isomorphic to $|M|$.

Lemma 1. $(|M|, w) \models \varphi$ iff $(M, w) \models \varphi$ for all $\varphi \in \mathcal{L}_{C A L}$.

Every pointed model $(M, w)$ is distinguished from all other non-bisimilar pointed models $(M, v)$ by some distinguishing formula $\delta_{w} \in \mathcal{L}_{E L}$.

Given a finite model $(M, w)$, distinguishing formula $\delta_{w}$ is constructed recursively as follows:

$$
\delta_{w}^{k+1}::=\delta_{w}^{0} \wedge \bigwedge_{a \in A}\left(\bigwedge_{w \sim a v} \widehat{K}_{a} \delta_{v}^{k} \wedge K_{a} \bigvee_{w \sim_{a} v} \delta_{v}^{k}\right),
$$

where $0 \leq k<|W|$, and $\delta_{w}^{0}$ is the conjunction of all literals that are true in $w$, i.e. $\delta_{w}^{0}::=\bigwedge_{w \in V(p)} p \wedge \bigwedge_{w \notin V(p)} \neg p$.

A distinguishing formula for a set of states $S$ is

$$
\delta_{S}::=\bigvee_{w \in S} \delta_{w}
$$

See Fig. 4, 5, 6, 7.

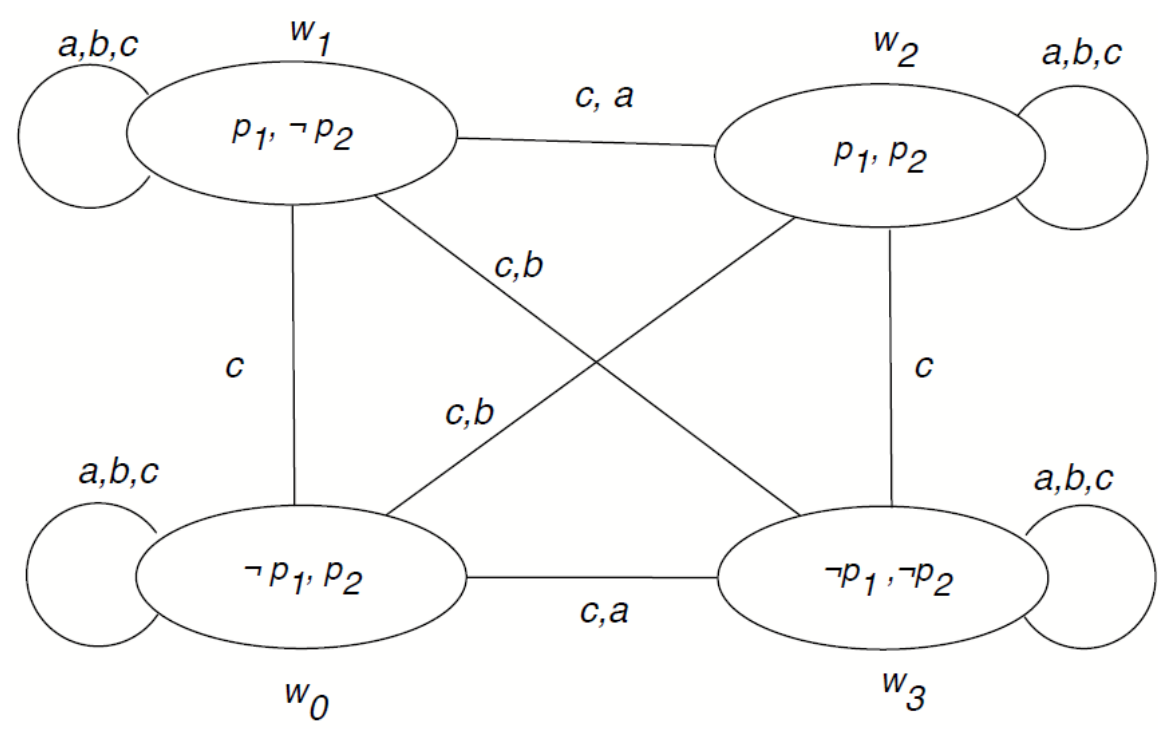

Fig. 4. Example 4 (Alternative model $M^{\prime}: a$ and $b$ only know "their" variable). 


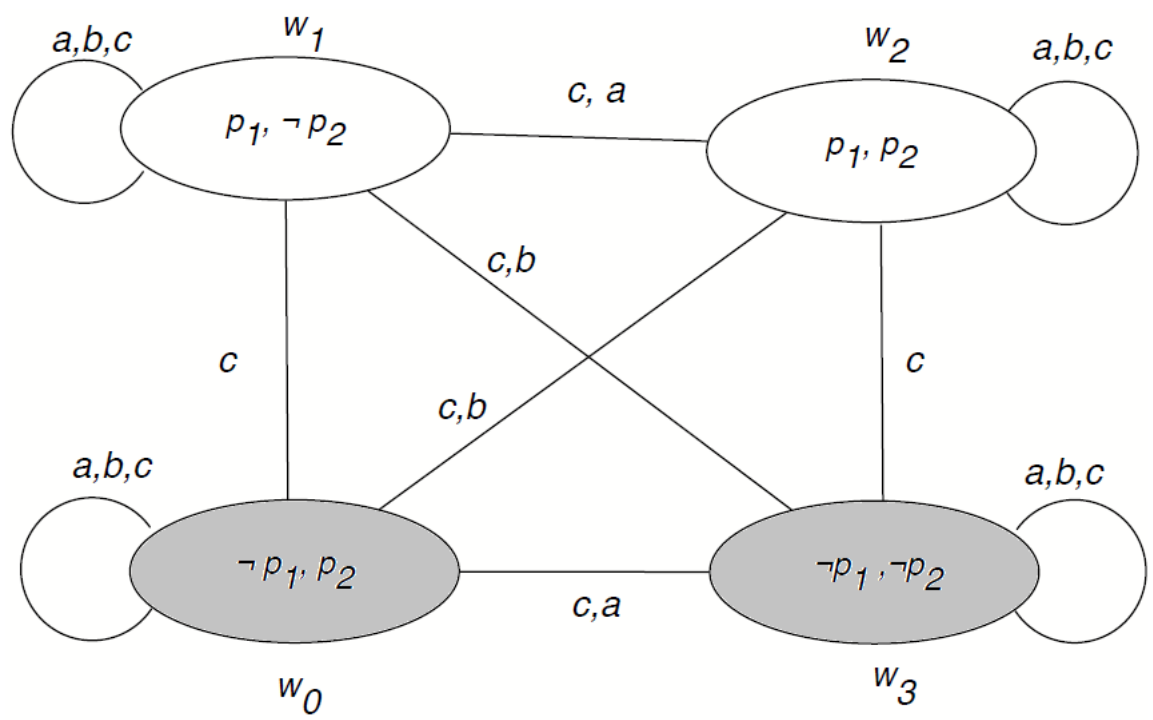

Fig. 5. Example 5 ( $a$ 's equivalence in $w_{0}$ : can announce $K_{a} \neg p_{1}$ ).

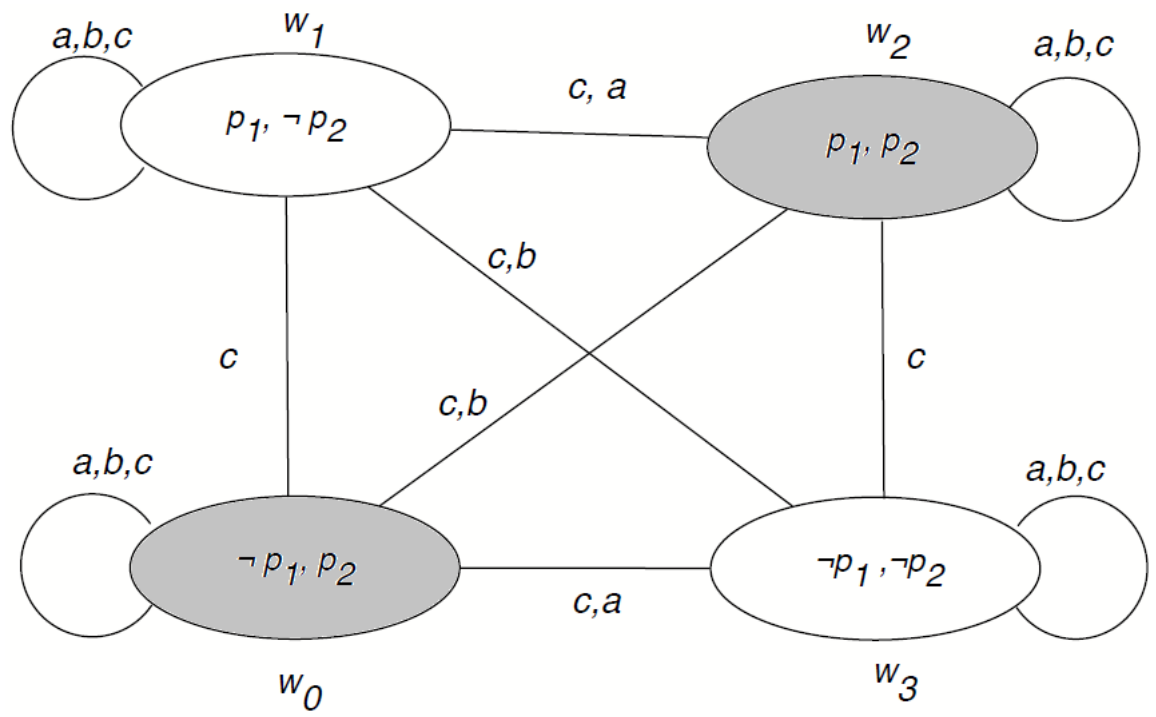

Fig. 6. Example 6 ( $b$ 's equivalence in $w_{0}$ : can announce $K_{b} p_{2}$ ).

Let $M / a=\left\{\left[w_{1}\right]_{a}, \ldots,\left[w_{n}\right]_{a}\right\}$ be the set of $a$-equivalence classes in $M$.

Definition 6. A strategy $X_{a}$ for an agent $a$ in a finite model $(M, w)$ is a union of equivalence classes of $a$ including $[w]_{a}$.

Definition 7. The set of all available strategies of $a$ is $S(a, w)=\left\{[w]_{a} \cup X_{a}\right.$ : $\left.X_{a} \subseteq \bigcup M / a\right\}$.

Definition 8. Group strategy $X_{G}$ is defined as $\cap_{a \in G} X_{a}$ for all $a \in G$. The set of available strategies for a group of agents $G$ is $S(G, w)=\left\{\cap_{a \in G} X_{a}: X_{a} \in\right.$ $S(a, w)\}$. 


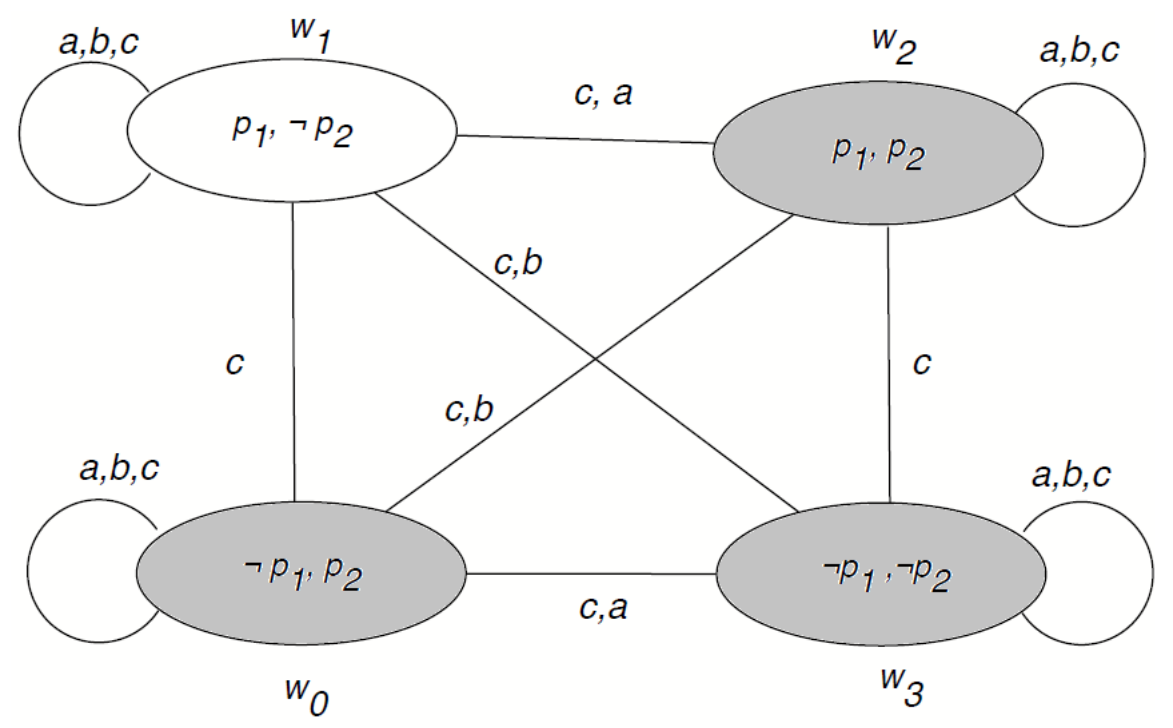

Fig. 7. Example 7 ( $w_{0}$ is the intersection of $a$ 's and $b$ 's equivalence classes in $w_{0}$ : can announce $K_{a} \neg p_{1}$ and $\left.K_{b} p_{2}\right)$.

Given a finite and bisimulation contracted model $(M, w)$ and strategy $X_{G}$, a distinguishing formula $\delta_{X_{G}}$ for $X_{G}$ is $\bigvee_{w \in X_{G}} \delta_{w}$.

In a bisimulation contracted model we propose alternative truth definition for $\langle[G]\rangle$,

$(M, w) \models\left\langle G \backslash \varphi\right.$ iff $\exists X_{G} \in S(G, w) \forall X_{A \backslash G} \in S(A \backslash G, w):(M, w)^{X_{G} \cap X_{A \backslash G}} \models \varphi$

\section{Model checking algorithm}

Algorithm $m c\left(M, w, \varphi_{0}\right)$, where $(M, w)$ is a pointed model and $\varphi_{0}$ is some formula:

case $\varphi_{0}$

$p:$ if $w \in V(p)$ then return true else return false;

$\neg \varphi$ : if $\neg m c(M, w, \varphi)$ then return true else return false;

$\varphi \wedge \psi:$ if $m c(M, w, \varphi)$ and $m c(M, w, \psi)$ then return true else return false;

$K_{a} \varphi:$ for all $v \sim_{a} w$

if $\neg m c(M, v, \varphi)$ then return false;

return true

$\langle\psi\rangle \varphi$ if $\neg m c(M, w, \psi)$ then return false, else compute the $\psi$-submodel of $M$ and return $m c\left(M^{\psi}, w, \varphi\right)$. 
$\langle G \backslash \varphi$ : compute $(|M|, w)$ and sets of strategies $S(G, w)$ and $S(A \backslash G, w)$

for all $X_{G} \in S(G, w)$

. check = true;

. for all $X_{A \backslash G} \in S(A \backslash G, w)$

. $\quad$ if $\neg m c\left(|M|^{X_{G} \cap X_{A \backslash G}}, w, \varphi\right)$ then check $=$ false

. $\quad$ if check then return true

return false.

Theorem 1. The model checking problem for CAL is PSPACE-complete.

\section{Summary}

We can use model checking to verify properties of announcements (for example, communication protocols, or data collection).

We can also use it to produce strategies (the right announcements to make) given the properties that should hold after the announcement

\section{Questions}

Question (V.I. Shalack): All these epistemic logic are S5, so we have got a problem with paradox of omniscience. Is there some another way to interpret knolwedge differently? For example, in jurisprudence and legal practice we are led by the principle that ignorance of law was no excuse, i.e. if a law was accepted, than everyone should know this laws and its consequences.

Answer: I prefer the syntactical interpretation of an epistemic modality, where every agent has his own 'knowledge set' of formulas, with their own rules how to infer consequences from these formulas. This way allows to model epistemic situations without logical omniscience. I agree that S5 is not always a good logic for modelling knowledge. On the other hand, if we work with a small number of formulas, then it is less paradoxical to assume omniscience limited to these formulas only.

Acknowledgements. Paper is RAS Institute of Philosophy Seminar Talk, 19 June 2018 (See video https://www. youtube.com/watch?v=Tfgg-Z04JkM). It is a joint work with Rustam Galimullin and Hans van Ditmarsch. This report is based on KI 2018 paper: Rustam Galimullin, Natasha Alechina and Hans van Ditmarsch. Model Checking for Coalition Announcement Logic. To appear in Proc. The 41st German Conference on Artificial Intelligence (KI 2018).

\section{References}

Plaza, 2007 - Plaza, J. "Logics of public communications", Synthese, 2007, Vol. 158, No. 2, pp. $165-179$.

Hintikka, 1962 - Hintikka, J. Knowledge and belief. An introduction to the logic of the two notions. Ithaca, New York: Cornell University Press, 1962. 179 pp. 
Ågotnes et all, 2010 - Ågotnes, T., Balbiani, P., van Ditmarsch, H., Seban, P. "Group announcement logic", Journal of Applied Logic, 2010, Vol. 8, No. 1, pp. 62-81.

Ågotnes, van Ditmarsch, 2008 - Ågotnes, T., van Ditmarsch, H. "Coalitions and announcements", in: Padgham, L., Parkes, D.C., Müller, J.P., Parsons, S. (eds.), 7th International Joint Conference on Autonomous Agents and Multiagent Systems (AAMAS 2008). IFAAMAS, 2008, pp. 673-680. 